\title{
Diagonalized Chebyshev Rational Spectral Methods for Second-Order Elliptic Problems on Unbounded Domains
}

\author{
Yanmin Ren, Xuhong Yu and Zhongqing Wang* \\ School of Science, University of Shanghai for Science and Technology, Shanghai, \\ 200093, P. R. China
}

Received 6 February 2018; Accepted (in revised version) 28 April 2018

\begin{abstract}
Diagonalized Chebyshev rational spectral methods for solving second-order elliptic problems on the half/whole line are proposed. Some Sobolev bi-orthogonal rational basis functions are constructed which lead to the diagonalization of discrete systems. Accordingly, both the exact solutions and the approximate solutions can be represented as infinite and truncated Fourier-like Chebyshev rational series. Numerical results demonstrate the effectiveness of the suggested approaches.
\end{abstract}

AMS subject classifications: 65N35, 41A20, 33C45, 35J15

Key words: Chebyshev rational spectral methods, Sobolev bi-orthogonal functions, second-order elliptic equations, numerical results.

\section{Introduction}

Many science and engineering problems are set on unbounded domains, such as fluid flows in an infinite strip, nonlinear wave equations in quantum mechanics and so on. How to accurately and efficiently solve such problems is a very important and difficult subject, since the unboundedness of the domain causes considerable theoretical and practical challenges. A variety of numerical approaches have been proposed and investigated for dealing with such problems. For spectral methods, we usually restrict calculations to some bounded subdomains and impose certain artificial boundary conditions. It is easy to be performed, but it lowers the accuracy sometimes. Alternatively, we may approximate the problems on unbounded domains directly by using some orthogonal polynomials/functions on unbounded domains, such as the Hermite spectral method and the Laguerre method, see, e.g., $[1,2,5,8,9,12,15,16,18-20,24]$. However, since the Laguerre and Hermite Gauss points are too concentrated near zero, the approximation results are usually not ideal, especially where the points are far away from zero. Another effective spectral method to approximate differential equations on unbounded domains is to use algebraically mapped

${ }^{*}$ Corresponding author. Email addresses: ym_ren@foxmail.com (Y. M. Ren), xhyu@usst.edu.cn (X. H. Yu), zqwang@usst.edu.cn (Z. Q. Wang) 
Legendre, Chebyshev or Jacobi functions, i.e., the so-called Legendre, Chebyshev or Jacobi rational spectral methods $[3,4,6,10,11,22,23]$. Compared with the first two method$\mathrm{s}$, we prefer the last one, since the distribution of the Gauss points is much reasonable. Accordingly, the numerical results would be better, especially for slow decay solutions.

As is well known, the utilization of Chebyshev rational functions usually leads to a sparse algebraic system (e.g., a nine-diagonal matrix for second-order problems on the whole line), the condition numbers increase as $\mathscr{O}\left(N^{2}\right)$ for second-order problems. However, in many cases, people still want to get a set of Fourier-like basis functions (see $[7,17]$ ), which are orthogonal or bi-orthogonal with respect to certain Sobolev inner product involving derivatives, such that the corresponding algebraic system is diagonal (see [21]).

Recently, Liu et al. $[13,14]$ constructed the Fourier-like Sobolev orthogonal basis functions based on generalized Laguerre functions, and applied them to second and fourth order elliptic equations on the half line. Motivated by [13,14,21], the main purpose of this paper is to construct the Fourier-like Chebyshev rational basis functions, which are biorthogonal with respect to certain Sobolev inner product. On this basis, we further propose the diagonalized Chebyshev rational spectral methods for second-order elliptic problems on the whole/half line.

The main advantages of the suggested algorithms include: (i). The exact solutions and the approximate solutions can be represented as infinite and truncated Fourier-like Chebyshev rational series, respectively; (ii) The condition numbers for the resulting algebraic systems are equal to 1 .

This paper is organized as follows. In Section 2, we introduce the Chebyshev rational functions on the whole/half line and their basic properties. In Section 3, we construct two kinds of Sobolev bi-orthogonal Chebyshev rational functions corresponding to the secondorder elliptic equations on the whole/half line, and propose the diagonalized Chebyshev rational spectral methods. Some numerical results are presented in Section 4 to demonstrate the effectiveness and accuracy.

\section{Notations and preliminaries}

Let $\Lambda$ be a certain interval and $\omega(x)$ be a weight function in the usual sense. For integer $r \geq 0$, we define the weighted Sobolev spaces $H_{\omega}^{r}(\Lambda)$ as usual, with the inner product $(u, v)_{r, \omega}$, the semi-norm $|v|_{r, \omega}$ and the norm $\|v\|_{r, \omega}$, respectively. We omit the subscript $r$ or $\omega(x)$ whenever $r=0$ or $\omega(x) \equiv 1$. For simplicity, we denote $\partial_{x}^{k} v=d^{k} v / d x^{k}$, $v^{\prime \prime}=d^{2} v / d x^{2}$ and $v^{\prime}=d v / d x$.

\subsection{Chebyshev polynomials}

We first recall the Chebyshev polynomials. Let $I=(-1,1)$ and $T_{k}(y)$ be the Chebyshev polynomial of degree $k$, which is the eigenfunction of the singular Strum-Liouville problem (cf. [20]):

$$
\sqrt{1-y^{2}} \partial_{y}\left(\sqrt{1-y^{2}} \partial_{y} T_{k}(y)\right)+k^{2} T_{k}(y)=0
$$


The set of all Chebyshev polynomials forms a complete $L_{\rho}^{2}(I)$-orthogonal system with the weight $\rho(y)=\frac{1}{\sqrt{1-y^{2}}}$, namely,

$$
\int_{I} T_{k}(y) T_{l}(y) \frac{1}{\sqrt{1-y^{2}}} d y=\frac{1}{2} \pi c_{k} \delta_{k, l},
$$

where $\delta_{k, l}$ is the Kronecker symbol, $c_{0}=2$ and $c_{k}=1$ for $k \geq 1$. By virtue of (2.1) and (2.2), we have

$$
\int_{I} \partial_{y} T_{k}(y) \partial_{y} T_{l}(y) \sqrt{1-y^{2}} d y=\frac{1}{2} \pi c_{k} k^{2} \delta_{k, l} .
$$

Moreover, the following recurrence relations are satisfied with $T_{0}(y)=1$ and $T_{1}(y)=y$ (cf. [20]),

$$
\begin{array}{ll}
T_{k+1}(y)=2 y T_{k}(y)-T_{k-1}(y), & k \geq 1, \\
2 T_{k}(y)=\frac{1}{k+1} \partial_{y} T_{k+1}(y)-\frac{1}{k-1} \partial_{y} T_{k-1}(y), & k \geq 2, \\
\left(1-y^{2}\right) \partial_{y} T_{k}(y)=\frac{k}{2} T_{k-1}(y)-\frac{k}{2} T_{k+1}(y), & k \geq 1, \\
\partial_{y} T_{k}(y)=2 k \sum_{i=0, i+k \text { odd }}^{k-1} \frac{1}{c_{i}} T_{i}(y) . &
\end{array}
$$

Particularly, $T_{k}(-y)=(-1)^{k} T_{k}(y), T_{k}( \pm 1)=( \pm 1)^{k}$ and $\partial_{y} T_{k}( \pm 1)=( \pm 1)^{k-1} k^{2}$.

\subsection{Chebyshev rational functions on the whole line}

We next recall the Chebyshev rational functions on the whole line. Let $\Lambda_{1}=(-\infty,+\infty)$. The Chebyshev rational function of degree $k$ on the whole line is defined by (cf. [22])

$$
R_{k}(x):=T_{k}\left(\frac{x}{\sqrt{x^{2}+1}}\right), \quad x \in \Lambda_{1}, \quad k \geq 0 .
$$

For convenience, let $R_{k}(x) \equiv 0$ for any integer $k<0$. By (2.1), we know that $R_{k}(x)$ is the eigenfunction of the singular Sturm-Liouville problem:

$$
\left(x^{2}+1\right) \partial_{x}\left(\left(x^{2}+1\right) \partial_{x} R_{k}(x)\right)+k^{2} R_{k}(x)=0, \quad x \in \Lambda_{1} .
$$

Due to (2.4), the Chebyshev rational functions satisfy the following recurrence relations with $R_{0}(x)=1, R_{1}(x)=\frac{x}{\sqrt{x^{2}+1}}$ (cf. [22]),

$$
\begin{array}{ll}
R_{k+1}(x)=\frac{2 x}{\sqrt{x^{2}+1}} R_{k}(x)-R_{k-1}(x), & k \geq 1, \\
2 R_{k}(x)=\left(x^{2}+1\right)^{\frac{3}{2}}\left(\frac{1}{k+1} \partial_{x} R_{k+1}(x)-\frac{1}{k-1} \partial_{x} R_{k-1}(x)\right), & k \geq 1, \\
\left(x^{2}+1\right)^{\frac{1}{2}} \partial_{x} R_{k}(x)=\frac{k}{2} R_{k-1}(x)-\frac{k}{2} R_{k+1}(x), & k \geq 1 .
\end{array}
$$


Next, let

$$
\omega(x)=\frac{1}{x^{2}+1}, \quad y=\frac{x}{\sqrt{x^{2}+1}} .
$$

Then, we have

$$
\frac{d y}{d x}=\left(x^{2}+1\right)^{-\frac{3}{2}}, \quad \frac{d x}{d y}=\left(1-y^{2}\right)^{-\frac{3}{2}}, \quad \omega(x) \frac{d x}{d y}=\rho(y) .
$$

The set of Chebyshev rational functions $\left\{R_{k}(x)\right\}_{k=0}^{\infty}$ forms an orthogonal system with the weight function $\omega(x)$ on the whole line, namely,

$$
\int_{\Lambda_{1}} R_{k}(x) R_{l}(x) \omega(x) d x=\frac{1}{2} \pi c_{k} \delta_{k, l} .
$$

By (2.6) and (2.10), we further know that

$$
\int_{\Lambda_{1}} \partial_{x} R_{k}(x) \partial_{x} R_{l}(x)\left(x^{2}+1\right) d x=\frac{1}{2} \pi c_{k} k^{2} \delta_{k, l} .
$$

Lemma 2.1. The following equalities hold:

$$
\begin{aligned}
& \partial_{x}^{2} R_{1}(x)=-\frac{3}{8} R_{1}(x)+\frac{9}{16} R_{3}(x)-\frac{3}{16} R_{5}(x), \\
& \partial_{x}^{2} R_{2}(x)=\frac{1}{2} R_{0}(x)-\frac{3}{2} R_{2}(x)+\frac{3}{2} R_{4}(x)-\frac{1}{2} R_{6}(x), \\
& \partial_{x}^{2} R_{3}(x)= \frac{21}{16} R_{1}(x)-\frac{27}{8} R_{3}(x)+3 R_{5}(x)-\frac{15}{16} R_{7}(x), \\
& \partial_{x}^{2} R_{k}(x)=-\frac{1}{16} k(k-2) R_{k-4}(x)+\frac{1}{4} k(k-1) R_{k-2}(x)-\frac{3}{8} k^{2} R_{k}(x) \\
& \quad+\frac{1}{4} k(k+1) R_{k+2}(x)-\frac{1}{16} k(k+2) R_{k+4}(x), \quad \forall k \geq 4 .
\end{aligned}
$$

Moreover,

$$
\begin{aligned}
& \partial_{x}^{2}\left(R_{0}(x) \omega(x)\right)=\left(R_{2}(x)-R_{4}(x)\right) \omega(x), \\
& \partial_{x}^{2}\left(R_{1}(x) \omega(x)\right)=\left(-\frac{3}{8} R_{1}(x)+\frac{21}{16} R_{3}(x)-\frac{15}{16} R_{5}(x)\right) \omega(x), \\
& \partial_{x}^{2}\left(R_{2}(x) \omega(x)\right)=\left(-\frac{3}{2} R_{2}(x)+3 R_{4}(x)-\frac{3}{2} R_{6}(x)\right) \omega(x), \\
& \partial_{x}^{2}\left(R_{3}(x) \omega(x)\right)=\left(\frac{9}{16} R_{1}(x)-\frac{27}{8} R_{3}(x)+5 R_{5}(x)-\frac{35}{16} R_{7}(x)\right) \omega(x), \\
& \partial_{x}^{2}\left(R_{k}(x) \omega(x)\right)=\left(-\frac{1}{16}(k-4)(k-2) R_{k-4}(x)+\frac{1}{4}(k-2)(k-1) R_{k-2}(x)\right. \\
& \quad-\frac{3}{8} k^{2} R_{k}(x)+\frac{1}{4}(k+2)(k+1) R_{k+2}(x) \\
&\left.\quad-\frac{1}{16}(k+4)(k+2) R_{k+4}(x)\right) \omega(x), \quad \forall k \geq 4 .
\end{aligned}
$$


Proof. By (2.7a), (2.7b), (2.7c) and a direct computation, we can verify easily the results of (2.12) and (2.13) for $0 \leq k \leq 3$. Next, by (2.7c) and (2.7b), we deduce that for $k \geq 4$,

$$
\begin{aligned}
& \partial_{x} R_{k}(x)=\frac{k}{2}\left(x^{2}+1\right)^{-\frac{1}{2}}\left(R_{k-1}(x)-R_{k+1}(x)\right) \\
= & \frac{k}{4}\left(x^{2}+1\right)\left(\frac{2}{k} \partial_{x} R_{k}(x)-\frac{1}{k-2} \partial_{x} R_{k-2}(x)-\frac{1}{k+2} \partial_{x} R_{k+2}(x)\right) \\
= & \left(x^{2}+1\right)\left(-\frac{k}{4(k-2)} \partial_{x} R_{k-2}(x)+\frac{1}{2} \partial_{x} R_{k}(x)-\frac{k}{4(k+2)} \partial_{x} R_{k+2}(x)\right) .
\end{aligned}
$$

Similarly, by using (2.7c), (2.7a) and (2.7b) successively, we get that for $l \geq 4$,

$$
\begin{aligned}
& \partial_{x}\left(R_{l}(x) \omega(x)\right)=\left(x^{2}+1\right)^{-1} \partial_{x} R_{l}(x)-2 x\left(x^{2}+1\right)^{-2} R_{l}(x) \\
= & \left(x^{2}+1\right)^{-\frac{3}{2}}\left(\frac{l-2}{2} R_{l-1}(x)-\frac{l+2}{2} R_{l+1}(x)\right) \\
= & -\frac{1}{4} \partial_{x} R_{l-2}(x)+\frac{1}{2} \partial_{x} R_{l}(x)-\frac{1}{4} \partial_{x} R_{l+2}(x) .
\end{aligned}
$$

Next, assume that

$$
\partial_{x}^{2} R_{k}(x)=\sum_{l=0}^{\infty} c_{k, l} R_{l}(x), \quad \partial_{x}^{2}\left(R_{k}(x) \omega(x)\right)=\sum_{l=0}^{\infty} d_{k, l} R_{l}(x) \omega(x) .
$$

Then, by (2.10) and integration by parts, we obtain

$$
\begin{aligned}
& c_{k, l}=\frac{\left(\partial_{x}^{2} R_{k}, R_{l}\right)_{\omega}}{\left(R_{l}, R_{l}\right)_{\omega}}=-\frac{\left(\partial_{x} R_{k}, \partial_{x}\left(R_{l} \omega\right)\right)}{\left(R_{l}, R_{l}\right)_{\omega}}=-\frac{2}{\pi c_{l}}\left(\partial_{x} R_{k}, \partial_{x}\left(R_{l} \omega\right)\right), \\
& d_{k, l}=\frac{\left(\partial_{x}^{2}\left(R_{k} \omega\right), R_{l}\right)}{\left(R_{l}, R_{l}\right)_{\omega}}=-\frac{\left(\partial_{x}\left(R_{k} \omega\right), \partial_{x} R_{l}\right)}{\left(R_{l}, R_{l}\right)_{\omega}}=-\frac{2}{\pi c_{l}}\left(\partial_{x}\left(R_{k} \omega\right), \partial_{x} R_{l}\right) .
\end{aligned}
$$

Moreover, by (2.14), (2.15) and (2.11), we deduce readily that

$$
\left(\partial_{x} R_{k}, \partial_{x}\left(R_{l} \omega\right)\right)= \begin{cases}\frac{3 k^{2} \pi}{16}, & l=k, \\ -\frac{k(k-1) \pi}{8}, & l=k-2, \\ -\frac{k(k+1) \pi}{8}, & l=k+2, \\ \frac{k(k-2) \pi}{32}, & l=k-4, \\ \frac{k(k+2) \pi}{32}, & l=k+4, \\ 0, & \text { otherwise. }\end{cases}
$$

A combination of (2.16)-(2.18) leads to the results of (2.12) and (2.13) for $k \geq 4$. 


\subsection{Chebyshev rational functions on the half line}

We now recall the Chebyshev rational functions on the half line. Let $\Lambda_{2}=(0,+\infty)$. The Chebyshev rational function of degree $k$ on the half line is defined by (cf. [11])

$$
r_{k}(x):=T_{k}\left(\frac{x-1}{x+1}\right), \quad x \in \Lambda_{2}, \quad k \geq 0 .
$$

For convenience, let $r_{k}(x) \equiv 0$ for any integer $k<0$. By (2.1) we know that $r_{k}(x)$ is the eigenfunction of the singular Sturm-Liouville problem:

$$
(x+1) \sqrt{x} \partial_{x}\left((x+1) \sqrt{x} \partial_{x} r_{k}(x)\right)+k^{2} r_{k}(x)=0, \quad x \in \Lambda_{2} .
$$

Due to (2.4), the Chebyshev rational functions on the half line satisfy the following recurrence relations with $r_{0}(x)=1, r_{1}(x)=\frac{x-1}{x+1}$,

$$
\begin{array}{ll}
r_{k+1}(x)=\frac{2(x-1)}{x+1} r_{k}(x)-r_{k-1}(x), & k \geq 1, \\
(x+1)^{2} r_{k}(x)=\frac{1}{k+1} \partial_{x} r_{k+1}(x)-\frac{1}{k-1} \partial_{x} r_{k-1}(x), & k \geq 2, \\
2 x \partial_{x} r_{k}(x)=\frac{k}{2} r_{k-1}(x)-\frac{k}{2} r_{k+1}(x), & k \geq 1 .
\end{array}
$$

Next, denote by

$$
\chi(x)=\frac{1}{(x+1) \sqrt{x}}, \quad y=\frac{x-1}{x+1}
$$

Then, we have

$$
\frac{d y}{d x}=\frac{2}{(x+1)^{2}}, \quad \frac{d x}{d y}=\frac{2}{(1-y)^{2}}, \quad \chi(x) \frac{d x}{d y}=\rho(y) .
$$

The set of Chebyshev rational functions $\left\{r_{k}(x)\right\}_{k=0}^{\infty}$ forms an orthogonal system with the weight function $\chi(x)$ on the half line, namely,

$$
\int_{\Lambda_{2}} r_{k}(x) r_{l}(x) \chi(x) d x=\frac{1}{2} \pi c_{k} \delta_{k, l} .
$$

Moreover, by (2.20) and (2.24), we find that

$$
\int_{\Lambda_{2}} \partial_{x} r_{k}(x) \partial_{x} r_{l}(x) \chi^{-1}(x) d x=\frac{1}{2} \pi c_{k} k^{2} \delta_{k, l} .
$$

In order to construct the diagonalized Chebyshev rational spectral methods for problems defined on the half line, we need to consider the following two kinds of polynomials:

$$
\begin{array}{lll}
p_{k}(x):=r_{k}(x)+\left(2-\delta_{k, 1}\right) r_{k-1}(x)+r_{k-2}(x), & & k \geq 1, \\
q_{k}(x):=r_{k}(x)+r_{k-1}(x), & & k \geq 1 .
\end{array}
$$

It is clear that $p_{k}(0)=q_{k}(0)=0$ for any $k \geq 1$. 
Lemma 2.2. For any $k \geq 1$, we have

$$
\begin{aligned}
\partial_{x}^{2} p_{k}(x)= & \frac{1}{16} k(k+1) r_{k+2}(x)-\frac{3}{8} k(k+1) r_{k+1}+\frac{3}{16}\left(5 k^{2}+7 k-2\right) r_{k}(x) \\
& -\frac{1}{4 c_{k-1}}\left(5 k^{2}+14 k-9\right) r_{k-1}(x)+\frac{1}{16}\left(15 k^{2}+111 k-96\left(\delta_{k, 2}+1\right)\right) r_{k-2}(x) \\
& -\frac{1}{8 c_{k-3}}\left(3 k^{2}+81 k-78\right) r_{k-3}(x)+\frac{1}{16}\left(k^{2}+187 k-186-288 \delta_{k, 4}\right) r_{k-4}(x) \\
& +12(k-1) \sum_{i=5}^{k} \frac{(-1)^{i}}{c_{k-i}} r_{k-i}(x) .
\end{aligned}
$$

Moreover, for $k, l \geq 1$, the following results hold:

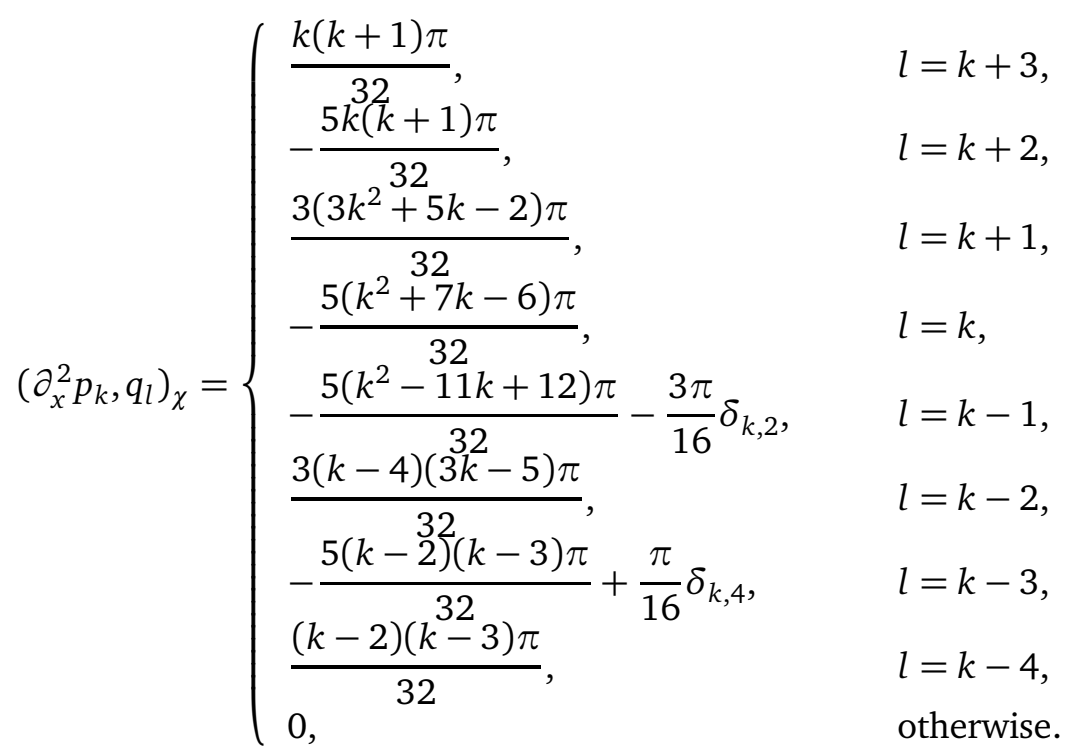

Proof. We first verify the result (2.27). Clearly, by (2.21a) we know that

$$
(x+1)^{-2} r_{k}(x)=\frac{1}{16} r_{k-2}(x)-\frac{1}{4} r_{k-1}(x)+\frac{3}{8} r_{k}(x)-\frac{1}{4} r_{k+1}(x)+\frac{1}{16} r_{k+2}(x), \quad k \geq 2 .
$$

Particularly,

$$
\begin{aligned}
& (x+1)^{-2} r_{0}(x)=\frac{3}{8} r_{0}(x)-\frac{1}{2} r_{1}(x)+\frac{1}{8} r_{2}(x), \\
& (x+1)^{-2} r_{1}(x)=-\frac{1}{4} r_{0}(x)+\frac{7}{16} r_{1}(x)-\frac{1}{4} r_{2}(x)+\frac{1}{16} r_{3}(x) .
\end{aligned}
$$


Next, by (2.4), (2.29) and (2.30) we get

$$
\begin{aligned}
\partial_{x} r_{0}(x) & =0, \quad \partial_{x} r_{1}(x)=\frac{1}{4} r_{2}(x)-r_{1}(x)+\frac{3}{4} r_{0}(x), \\
\partial_{x} r_{k}(x) & =\left(2 k \sum_{i=0, i+k \text { odd }}^{k-1} \frac{1}{c_{i}} r_{i}(x)\right) \frac{2}{(x+1)^{2}} \\
& =\frac{1}{4} k r_{k+1}(x)-k r_{k}(x)+\frac{7}{4} k r_{k-1}(x)-2 k \sum_{i=0}^{k-2} \frac{(-1)^{i}}{c_{i}} r_{i}(x), \quad k \geq 2 .
\end{aligned}
$$

From (2.31) we further deduce that

$$
\begin{aligned}
\partial_{x}^{2} r_{k}(x)=\frac{1}{16} & k(k+1) r_{k+2}(x)-\frac{1}{4} k(2 k+1) r_{k+1}(x)+\frac{15}{8} k^{2} r_{k}(x) \\
& -\left(\frac{9}{4} k(2 k-1)-\delta_{k, 1}\right) r_{k-1}(x)+\left(\frac{129}{32} k(2 k-2)-8 \delta_{k, 2}\right) r_{k-2}(x) \\
& +2 k \sum_{i=3}^{k} \frac{(-1)^{i} i(2 k-i)}{c_{k-i}} r_{k-i}(x) .
\end{aligned}
$$

Hence

$$
\begin{aligned}
\partial_{x}^{2} p_{k}(x)= & \partial_{x}^{2} r_{k}(x)+2 \partial_{x}^{2} r_{k-1}(x)+\partial_{x}^{2} r_{k-2}(x) \\
= & \frac{1}{16} k(k+1) r_{k+2}(x)-\frac{3}{8} k(k+1) r_{k+1}+\frac{3}{16}\left(5 k^{2}+7 k-2\right) r_{k}(x) \\
& -\frac{1}{4 c_{k-1}}\left(5 k^{2}+14 k-9\right) r_{k-1}(x)+\frac{1}{16}\left(15 k^{2}+111 k-96\left(\delta_{k, 2}+1\right)\right) r_{k-2}(x) \\
& -\frac{1}{8 c_{k-3}}\left(3 k^{2}+81 k-78\right) r_{k-3}(x)+\frac{1}{16}\left(k^{2}+187 k-186-288 \delta_{k, 4}\right) r_{k-4}(x) \\
& +12(k-1) \sum_{i=5}^{k} \frac{(-1)^{i}}{c_{k-i}} r_{k-i}(x) .
\end{aligned}
$$

This ends the proof of (2.27).

Next, by (2.24) and (2.33) we get that for $l<k-4$,

$$
\left(\partial_{x}^{2} p_{k}, q_{l}\right)_{\chi}=\left(\partial_{x}^{2} p_{k}, r_{l}+r_{l-1}\right)_{\chi}=12(k-1) \sum_{i=5}^{k} \frac{(-1)^{i}}{c_{k-i}}\left(r_{k-i}, r_{l}+r_{l-1}\right)_{\chi}=0
$$

Similarly, we have $\left(\partial_{x}^{2} p_{k}, q_{l}\right)_{\chi}=0$ for $l>k+3$. Moreover, by (2.24) and (2.33), a direct 
calculation yields

$$
\begin{aligned}
& \left(\partial_{x}^{2} p_{k}, q_{k+3}\right)_{\chi}=\frac{k(k+1) \pi}{32}, \quad\left(\partial_{x}^{2} p_{k}, q_{k+2}\right)_{\chi}=-\frac{5 k(k+1) \pi}{32} \\
& \left(\partial_{x}^{2} p_{k}, q_{k+1}\right)_{\chi}=\frac{3\left(3 k^{2}+5 k-2\right) \pi}{32}, \quad\left(\partial_{x}^{2} p_{k}, q_{k}\right)_{\chi}=-\frac{5\left(k^{2}+7 k-6\right) \pi}{32} \\
& \left(\partial_{x}^{2} p_{k}, q_{k-1}\right)_{\chi}=-\frac{5\left(k^{2}-11 k+12\right) \pi}{32}-\frac{3 \pi}{16} \delta_{k, 2}, \\
& \left(\partial_{x}^{2} p_{k}, q_{k-2}\right)_{\chi}=\frac{3(k-4)(3 k-5) \pi}{32}, \\
& \left(\partial_{x}^{2} p_{k}, q_{k-3}\right)_{\chi}=-\frac{5(k-2)(k-3) \pi}{32}+\frac{\pi}{16} \delta_{k, 4}, \\
& \left(\partial_{x}^{2} p_{k}, q_{k-4}\right)_{\chi}=\frac{(k-2)(k-3) \pi}{32} .
\end{aligned}
$$

This leads to the result of (2.28).

\section{Diagonalized Chebyshev rational spectral methods}

In this section, we propose diagonalized Chebyshev rational spectral methods for solving second-order elliptic equations on unbounded domains. The main idea is to find biorthogonal rational functions with respect to the coercive bilinear form, such that both the exact solution and the approximate solution can be expressed explicitly.

\subsection{Diagonalized Chebyshev rational spectral method on the whole line}

Consider the second-order elliptic equation on the whole line:

$$
\left\{\begin{array}{l}
-u^{\prime \prime}(x)+\mu u(x)=f(x), \quad \mu \geq 0, \quad x \in \Lambda_{1}, \\
\lim _{|x| \rightarrow \infty} x^{-\frac{1}{2}} u(x)=0 .
\end{array}\right.
$$

A weak formulation of (3.1) is to find $u \in H_{\omega}^{1}\left(\Lambda_{1}\right)$ such that

$$
A_{\mu}(u, v):=\left(\partial_{x} u, \partial_{x}(v \omega)\right)+\mu(u, v)_{\omega}=(f, v)_{\omega}, \quad \forall v \in H_{\omega}^{1}\left(\Lambda_{1}\right) .
$$

Next, let $N$ be any positive integer, and $\mathscr{R}_{N}\left(\Lambda_{1}\right)=\operatorname{span}\left\{R_{0}(x), R_{1}(x), \cdots, R_{N}(x)\right\}$. The Chebyshev rational spectral scheme for (3.2) is to find $u_{N} \in \mathscr{R}_{N}\left(\Lambda_{1}\right)$ such that

$$
A_{\mu}\left(u_{N}, \phi\right)=(f, \phi)_{\omega}, \quad \forall \phi \in \mathscr{R}_{N}\left(\Lambda_{1}\right) .
$$

To propose a diagonalized approximation scheme for (3.3), we need to construct two kinds of basis functions $\left\{\varphi_{k}\right\}_{0 \leq k \leq N}$ and $\left\{\psi_{k}\right\}_{0 \leq k \leq N}$, which are bi-orthogonal with respect to the bilinear operator $A_{\mu}(\cdot, \cdot)$. 
Lemma 3.1. Let $\varphi_{k}, \psi_{k} \in \mathscr{R}_{k}\left(\Lambda_{1}\right)$ be the bi-orthogonal Chebyshev rational functions such that $\varphi_{k}-R_{k} \in \mathscr{R}_{k-1}\left(\Lambda_{1}\right), \psi_{k}-R_{k} \in \mathscr{R}_{k-1}\left(\Lambda_{1}\right)$ and

$$
A_{\mu}\left(\varphi_{k}, \psi_{l}\right)=\eta_{k} \delta_{k, l}, \quad \forall k, l \geq 0
$$

Then we have

$$
\begin{aligned}
& \varphi_{k}(x)=R_{k}(x)+a_{k} \varphi_{k-2}(x)+b_{k} \varphi_{k-4}(x), \\
& \psi_{k}(x)=R_{k}(x)+c_{k} \psi_{k-2}(x)+d_{k} \psi_{k-4}(x),
\end{aligned}
$$

where $\varphi_{k}(x)=\psi_{k}(x) \equiv 0$ for $k<0, \eta_{k}=0$ for $k<0, a_{k}=c_{k}=0$ for $k<2, b_{k}=d_{k}=0$ for $k<4$, and

$$
\begin{aligned}
& \eta_{0}=\mu \pi, \quad \eta_{k}=\frac{\pi}{2}\left(\mu+\frac{3 k^{2}}{8}\right)-a_{k} c_{k} \eta_{k-2}-b_{k} d_{k} \eta_{k-4}, \\
& a_{2}=\frac{\pi}{2 \eta_{0}}, \quad a_{3}=\frac{21 \pi}{32 \eta_{1}}, \quad a_{4}=\frac{3 \pi}{2 \eta_{2}}, \quad b_{4}=-\frac{\pi}{2 \eta_{0}}, \\
& c_{2}=0, \quad c_{3}=\frac{9 \pi}{32 \eta_{1}}, \quad c_{4}=\frac{3 \pi}{4 \eta_{2}}, \quad d_{4}=0, \\
& a_{k}=\frac{k(k-1) \pi}{8 \eta_{k-2}}-\frac{k(k-2) \pi c_{k-2}}{32 \eta_{k-2}}, \quad b_{k}=-\frac{k(k-2) \pi}{32 \eta_{k-4}}, \quad \forall k \geq 5, \\
& c_{k}=\frac{(k-2)(k-1) \pi}{8 \eta_{k-2}}-\frac{(k-4)(k-2) \pi a_{k-2}}{32 \eta_{k-2}}, \\
& d_{k}=-\frac{(k-4)(k-2) \pi}{32 \eta_{k-4}}, \quad \forall k \geq 5 .
\end{aligned}
$$

Proof. Let

$$
\varphi_{k}(x)=R_{k}(x)+\sum_{i=0}^{k-1} a_{k, i} \varphi_{i}(x), \quad \psi_{k}(x)=R_{k}(x)+\sum_{i=0}^{k-1} c_{k, i} \psi_{i}(x)
$$

We first use mathematical induction to verify (3.5). By (3.7), (2.12), (2.13) and (2.10), we deduce that

$$
\begin{aligned}
& A_{\mu}\left(\varphi_{1}, \psi_{0}\right)=A_{\mu}\left(R_{1}, \psi_{0}\right)+a_{1,0} A_{\mu}\left(\varphi_{0}, \psi_{0}\right)=-\left(\partial_{x}^{2} R_{1}, R_{0}\right)_{\omega}+a_{1,0} \eta_{0}=a_{1,0} \eta_{0}, \\
& A_{\mu}\left(\varphi_{0}, \psi_{1}\right)=A_{\mu}\left(\varphi_{0}, R_{1}\right)+c_{1,0} A_{\mu}\left(\varphi_{0}, \psi_{0}\right)=-\left(R_{0}, \partial_{x}^{2}\left(R_{1} \omega\right)\right)+c_{1,0} \eta_{0}=c_{1,0} \eta_{0} .
\end{aligned}
$$

On the other hand, by (3.4) we know that $A_{\mu}\left(\varphi_{1}, \psi_{0}\right)=A_{\mu}\left(\varphi_{0}, \psi_{1}\right)=0$. This means $a_{1,0}=c_{1,0}=0$ and $\varphi_{1}(x)=\psi_{1}(x)=R_{1}(x)$. Similarly, by (3.4), (2.12), (2.13) and (2.10), 
we have

$$
\begin{aligned}
A_{\mu}\left(\varphi_{2}, \psi_{0}\right) & =A_{\mu}\left(R_{2}, \psi_{0}\right)+a_{2,0} A_{\mu}\left(\varphi_{0}, \psi_{0}\right)+a_{2,1} A_{\mu}\left(\varphi_{1}, \psi_{0}\right) \\
& =-\left(\partial_{x}^{2} R_{2}, R_{0}\right)_{\omega}+a_{2,0} \eta_{0}=-\frac{\pi}{2}+a_{2,0} \eta_{0}=0, \\
A_{\mu}\left(\varphi_{2}, \psi_{1}\right) & =A_{\mu}\left(R_{2}, \psi_{1}\right)+a_{2,0} A_{\mu}\left(\varphi_{0}, \psi_{1}\right)+a_{2,1} A_{\mu}\left(\varphi_{1}, \psi_{1}\right) \\
& =-\left(\partial_{x}^{2} R_{2}, R_{1}\right)_{\omega}+a_{2,1} \eta_{1}=a_{2,1} \eta_{1}=0, \\
A_{\mu}\left(\varphi_{0}, \psi_{2}\right) & =A_{\mu}\left(\varphi_{0}, R_{2}\right)+c_{2,0} A_{\mu}\left(\varphi_{0}, \psi_{0}\right)+c_{2,1} A_{\mu}\left(\varphi_{0}, \psi_{1}\right) \\
& =-\left(R_{0}, \partial_{x}^{2}\left(R_{2} \omega\right)\right)+c_{2,0} \eta_{0}=c_{2,0} \eta_{0}=0, \\
A_{\mu}\left(\varphi_{1}, \psi_{2}\right) & =A_{\mu}\left(\varphi_{1}, R_{2}\right)+c_{2,0} A_{\mu}\left(\varphi_{1}, \psi_{0}\right)+c_{2,1} A_{\mu}\left(\varphi_{1}, \psi_{1}\right) \\
& =-\left(R_{1}, \partial_{x}^{2}\left(R_{2} \omega\right)\right)+c_{2,1} \eta_{1}=c_{2,1} \eta_{1}=0 .
\end{aligned}
$$

Thereby, $a_{2,0}=\frac{\pi}{2 \eta_{0}}, a_{2,1}=c_{2,0}=c_{2,1}=0$. Accordingly,

$$
\varphi_{2}(x)=R_{2}(x)+a_{2,0} \varphi_{0}(x), \quad \psi_{2}(x)=R_{2}(x) .
$$

In the same manner, we can verify the results of (3.5), as well as the corresponding coefficients in (3.6) for $k=3,4$. Next, assume that for any $0 \leq l \leq k-1$ and $k \geq 5$,

$$
\begin{aligned}
& \varphi_{l}(x)=R_{l}(x)+a_{l, l-2} \varphi_{l-2}(x)+a_{l, l-4} \varphi_{l-4}(x), \\
& \psi_{l}(x)=R_{l}(x)+c_{l, l-2} \psi_{l-2}(x)+c_{l, l-4} \psi_{l-4}(x) .
\end{aligned}
$$

We shall prove that for $k \geq 5$,

$$
\begin{aligned}
& \varphi_{k}(x)=R_{k}(x)+a_{k, k-2} \varphi_{k-2}(x)+a_{k, k-4} \varphi_{k-4}(x), \\
& \psi_{k}(x)=R_{k}(x)+c_{k, k-2} \psi_{k-2}(x)+c_{k, k-4} \psi_{k-4}(x) .
\end{aligned}
$$

Clearly, by (3.4), (3.7) and (2.10), we get that for $k>l \geq 0$,

$$
\begin{aligned}
& A_{\mu}\left(\varphi_{k}, \psi_{l}\right)=A_{\mu}\left(R_{k}, \psi_{l}\right)+\sum_{i=0}^{k-1} a_{k, i} A_{\mu}\left(\varphi_{i}, \psi_{l}\right)=-\left(\partial_{x}^{2} R_{k}, \psi_{l}\right)_{\omega}+a_{k, l} \eta_{l}=0, \\
& A_{\mu}\left(\varphi_{l}, \psi_{k}\right)=A_{\mu}\left(\varphi_{l}, R_{k}\right)+\sum_{i=0}^{k-1} c_{k, i} A_{\mu}\left(\varphi_{l}, \psi_{i}\right)=-\left(\varphi_{l}, \partial_{x}^{2}\left(R_{k} \omega\right)\right)+c_{k, l} \eta_{l}=0 .
\end{aligned}
$$

Taking $l=0,1, \cdots, k-5, l=k-3$ and $l=k-1$ in (3.9) successively, and using (2.12), (2.13), (2.10) and the induction assumption, we derive readily that for $k \geq 5$,

$$
a_{k, l}=c_{k, l}=0, \quad \forall 0 \leq l \leq k-5 \text { or } l=k-3, k-1 .
$$

This leads to (3.8). For simplicity of notations, we take $a_{k}:=a_{k, k-2}, b_{k}:=a_{k, k-4}, c_{k}:=$ $c_{k, k-2}$ and $d_{k}:=c_{k, k-4}$ in (3.8), then we obtain the result (3.5). 
It remains to confirm the coefficients $a_{k}, b_{k}, c_{k}, d_{k}$ and $\eta_{k}$. By using (3.9), (3.5), (2.12), (2.13) and (2.10), we get that for $k \geq 5$,

$$
\begin{aligned}
a_{k} & =\frac{1}{\eta_{k-2}}\left(\partial_{x}^{2} R_{k}, \psi_{k-2}\right)_{\omega}=\frac{k(k-1)}{4 \eta_{k-2}}\left(R_{k-2}, R_{k-2}\right)_{\omega}-\frac{k(k-2) c_{k-2}}{16 \eta_{k-2}}\left(R_{k-4}, R_{k-4}\right)_{\omega} \\
& =\frac{k(k-1) \pi}{8 \eta_{k-2}}-\frac{k(k-2) \pi c_{k-2}}{32 \eta_{k-2}}, \\
c_{k} & =\frac{1}{\eta_{k-2}}\left(\varphi_{k-2}, \partial_{x}^{2}\left(R_{k} \omega\right)\right) \\
& =\frac{(k-2)(k-1)}{4 \eta_{k-2}}\left(R_{k-2}, R_{k-2}\right)-\frac{(k-4)(k-2) a_{k-2}}{16 \eta_{k-2}}\left(R_{k-4}, R_{k-4}\right)_{\omega} \\
& =\frac{(k-2)(k-1) \pi}{8 \eta_{k-2}}-\frac{(k-4)(k-2) \pi a_{k-2}}{32 \eta_{k-2}}, \\
b_{k} & =\frac{1}{\eta_{k-4}}\left(\partial_{x}^{2} R_{k}, \psi_{k-4}\right)_{\omega}=-\frac{k(k-2)}{16 \eta_{k-4}}\left(R_{k-4}, R_{k-4}\right)_{\omega}=-\frac{k(k-2) \pi}{32 \eta_{k-4}}, \\
d_{k} & =\frac{1}{\eta_{k-4}}\left(\varphi_{k-4}, \partial_{x}^{2}\left(R_{k} \omega\right)\right)=-\frac{(k-4)(k-2)}{16 \eta_{k-4}}\left(R_{k-4}, R_{k-4}\right)_{\omega}=-\frac{(k-4)(k-2) \pi}{32 \eta_{k-4}} .
\end{aligned}
$$

Next, by (3.2), (2.13) and (2.10), we derive that

$$
\begin{aligned}
\eta_{0} & =A_{\mu}\left(\varphi_{0}, \psi_{0}\right)=\left(\partial_{x} \varphi_{0}, \partial_{x}\left(\psi_{0} \omega\right)\right)+\mu\left(\varphi_{0}, \psi_{0}\right)_{\omega} \\
& =\left(\partial_{x} R_{0}, \partial_{x}\left(R_{0} \omega\right)\right)+\mu\left(R_{0}, R_{0}\right)_{\omega}=\mu \pi
\end{aligned}
$$

By using (3.4) and (3.5), we know that for $k \geq 1$,

$$
\begin{aligned}
A_{\mu}\left(R_{k}, R_{k}\right) & =A_{\mu}\left(\varphi_{k}-a_{k} \varphi_{k-2}-b_{k} \varphi_{k-4}, \psi_{k}-c_{k} \psi_{k-2}-d_{k} \psi_{k-4}\right) \\
& =A_{\mu}\left(\varphi_{k}, \psi_{k}\right)+a_{k} c_{k} A_{\mu}\left(\varphi_{k-2}, \psi_{k-2}\right)+b_{k} d_{k} A_{\mu}\left(\varphi_{k-4}, \psi_{k-4}\right) \\
& =\eta_{k}+a_{k} c_{k} \eta_{k-2}+b_{k} d_{k} \eta_{k-4} .
\end{aligned}
$$

On the other hand, by (2.12), (2.10) and the definition of $A_{\mu}(\cdot, \cdot)$, we have

$$
\begin{aligned}
A_{\mu}\left(R_{k}, R_{k}\right) & =-\left(\partial_{x}^{2} R_{k}, R_{k}\right)_{\omega}+\mu\left(R_{k}, R_{k}\right)_{\omega} \\
& =\frac{3 k^{2}}{8}\left(R_{k}, R_{k}\right)+\mu\left(R_{k}, R_{k}\right)_{\omega}=\frac{\pi}{2}\left(\mu+\frac{3 k^{2}}{8}\right) .
\end{aligned}
$$

Therefore

$$
\eta_{k}=\frac{\pi}{2}\left(\mu+\frac{3 k^{2}}{8}\right)-a_{k} c_{k} \eta_{k-2}-b_{k} d_{k} \eta_{k-4}, \quad k \geq 1 .
$$

This ends the proof.

Obviously, $\mathscr{R}_{N}\left(\Lambda_{1}\right)=\left\{\varphi_{k}(x): 0 \leq k \leq N\right\}$. Thus the variational forms (3.2) and (3.3) together with the biorthogonality of $\left\{\varphi_{k}(x)\right\}$ and $\left\{\psi_{k}(x)\right\}$ lead to the following main theorem in this subsection. 
Theorem 3.1. Let $u(x)$ and $u_{N}(x)$ be the solutions of (3.2) and (3.3), respectively. Then both $u(x)$ and $u_{N}(x)$ have the explicit representations in $\left\{\varphi_{k}(x)\right\}$,

$$
\begin{aligned}
& u(x)=\sum_{k=0}^{\infty} \hat{u}_{k} \varphi_{k}(x), \quad u_{N}(x)=\sum_{k=0}^{N} \hat{u}_{k} \varphi_{k}(x), \\
& \hat{u}_{k}=\frac{1}{\eta_{k}} A_{\mu}\left(u, \psi_{k}\right)=\frac{1}{\eta_{k}}\left(f, \psi_{k}\right)_{\omega}, \quad k \geq 0 .
\end{aligned}
$$

Remark 3.1. Wang and Guo [22] presented the convergence of scheme (3.3): if $\mu>\frac{1}{4}$ and $r \geq 1$, then

$$
\left\|u-u_{N}\right\|_{1, \omega} \leq c N^{1-r}\left(\sum_{k=0}^{r}\left\|\left(x^{2}+1\right)^{r / 2+k / 2-1 / 2} \partial_{x}^{k} u\right\|_{\omega}^{2}\right)^{1 / 2} .
$$

\subsection{Diagonalized Chebyshev rational spectral method on the half line}

Consider the second-order elliptic equation on the half line:

$$
\begin{cases}-u^{\prime \prime}(x)+\mu u(x)=f(x), & \mu \geq 0, \quad x \in \Lambda_{2}, \\ u(0)=0, & \lim _{x \rightarrow+\infty} x^{-\frac{1}{4}} u(x)=0 .\end{cases}
$$

Let $H_{0, \chi}^{1}\left(\Lambda_{2}\right)=\left\{v \in H_{\chi}^{1}\left(\Lambda_{2}\right): v(0)=0\right\}$. A weak formulation of (3.10) is to find $u \in$ $H_{0, \chi}^{1}\left(\Lambda_{2}\right)$ such that

$$
B_{\mu}(u, v):=\left(\partial_{\chi} u, \partial_{x}(v \chi)\right)+\mu(u, v)_{\chi}=(f, v)_{\chi}, \quad \forall v \in H_{0, \chi}^{1}\left(\Lambda_{2}\right) .
$$

Denote $\mathscr{R}_{N}^{0}\left(\Lambda_{2}\right)=\operatorname{span}\left\{r_{0}(x), r_{1}(x), \cdots, r_{N}(x)\right\} \cap H_{0, \chi}^{1}\left(\Lambda_{2}\right)$. The Chebyshev rational spectral scheme for (3.11) is to find $u_{N} \in \mathscr{R}_{N}^{0}\left(\Lambda_{2}\right)$ such that

$$
B_{\mu}\left(u_{N}, \phi\right)=(f, \phi)_{\chi}, \quad \forall \phi \in \mathscr{R}_{N}^{0}\left(\Lambda_{2}\right) .
$$

To propose a diagonalized approximation scheme for (3.12), we need to construct two kinds of basis functions $\left\{\Phi_{k}(x)\right\}_{1 \leq k \leq N}$ and $\left\{\Psi_{k}(x)\right\}_{1 \leq k \leq N}$, which are bi-orthogonal with respect to the bilinear operator $B_{\mu}(\cdot, \cdot)$.

Lemma 3.2. Let $p_{k}(x)=q_{k}(x) \equiv 0$ for any $k \leq 0$, and

$$
\Phi_{1}(x):=p_{1}(x)=r_{1}(x)+r_{0}(x) \in \mathscr{R}_{1}^{0}\left(\Lambda_{2}\right), \quad \Psi_{1}(x):=q_{1}(x)=r_{1}(x)+r_{0}(x) \in \mathscr{R}_{1}^{0}\left(\Lambda_{2}\right) .
$$

Assume that $\Phi_{k}, \Psi_{k} \in \mathscr{R}_{k}^{0}\left(\Lambda_{2}\right)$ are the bi-orthogonal Chebyshev rational functions such that $\Phi_{k}-p_{k} \in \mathscr{R}_{k-1}^{0}\left(\Lambda_{2}\right), \Psi_{k}-q_{k} \in \mathscr{R}_{k-1}^{0}\left(\Lambda_{2}\right)$ and

$$
B_{\mu}\left(\Phi_{k}, \Psi_{l}\right)=\rho_{k} \delta_{k, l}, \quad \forall k, l \geq 1 .
$$


Then for $k \geq 2$, we have

$$
\begin{aligned}
& \Phi_{k}(x)=p_{k}(x)+a_{k-1} \Phi_{k-1}(x)+b_{k-2} \Phi_{k-2}(x)+c_{k-3} \Phi_{k-3}(x)+d_{k-4} \Phi_{k-4}(x), \\
& \Psi_{k}(x)=q_{k}(x)+e_{k-1} \Psi_{k-1}(x)+h_{k-2} \Psi_{k-2}(x)+m_{k-3} \Psi_{k-3}(x),
\end{aligned}
$$

where $\Phi_{k}(x)=\Psi_{k}(x) \equiv 0$ for $k \leq 0, \rho_{k}=a_{k}=b_{k}=c_{k}=d_{k}=e_{k}=h_{k}=m_{k}=0$ for $k \leq 0$, and

$$
\begin{aligned}
a_{1}=- & \frac{(8 \mu-3) \pi}{4 \rho_{1}}, \\
a_{k-1}=- & \frac{5\left(k^{2}-11 k+12\right) \pi+48 \mu \pi}{32 \rho_{k-1}} \\
& \quad+\frac{1}{\rho_{k-1}}\left(b_{k-2} e_{k-2} \rho_{k-2}+c_{k-3} h_{k-3} \rho_{k-3}+d_{k-4} m_{k-4} \rho_{k-4}\right), \quad k \geq 3, \\
b_{k-2}= & \frac{3(k-4)(3 k-5) \pi-16 \mu \pi}{32 \rho_{k-2}} \\
& \quad+\frac{1}{\rho_{k-2}}\left(c_{k-3} e_{k-3} \rho_{k-3}+d_{k-4} h_{k-4} \rho_{k-4}\right), \quad k \geq 3, \\
c_{k-3}=- & \frac{5(k-2)(k-3) \pi}{32 \rho_{k-3}}+\frac{1}{16 \rho_{k-3}} \pi \delta_{k, 4}+\frac{1}{\rho_{k-3}} d_{k-4} e_{k-4} \rho_{k-4}, \quad k \geq 4, \\
d_{k-4}= & \frac{(k-2)(k-3) \pi}{32 \rho_{k-4}}, \quad k \geq 5, \\
e_{k-1}= & \frac{3\left(3 k^{2}-k-4\right) \pi-16 \mu \pi}{32 \rho_{k-1}} \\
& +\frac{1}{\rho_{k-1}}\left(a_{k-2} h_{k-2} \rho_{k-2}+b_{k-3} m_{k-3} \rho_{k-3}\right), \quad k \geq 2, \\
h_{k-2}= & -\frac{5 k-2(k-1) \pi}{32 \rho_{k-2}}+\frac{1}{\rho_{k-2}} a_{k-3} m_{k-3} \rho_{k-3}, \quad k \geq 3, \\
m_{k-3}= & \frac{(k-3)(k-2) \pi}{32 \rho_{k-3}}, \quad k \geq 4, \\
\rho_{k}= & \frac{5\left(k^{2}+7 k-6\right) \pi+48 \mu \pi}{32}-a_{k-1} e_{k-1} \rho_{k-1}-b_{k-2} \rho_{k-3}, \quad k \geq 1 .
\end{aligned}
$$

Proof. Let

$$
\Phi_{k}(x)=p_{k}(x)+\sum_{i=1}^{k-1} a_{k, i} \Phi_{i}(x), \quad \Psi_{k}(x)=q_{k}(x)+\sum_{i=1}^{k-1} b_{k, i} \Psi_{i}(x), \quad k \geq 2 .
$$

Then, by (3.16), (2.28), (2.26) and (2.24), we deduce that for any $1 \leq l \leq k-5$,

$$
B_{\mu}\left(p_{k}, \Psi_{l}\right)=\left(\partial_{x} p_{k}, \partial_{x}\left(\Psi_{l} \chi\right)\right)+\mu\left(p_{k}, \Psi_{l}\right)_{\chi}=-\left(\partial_{x}^{2} p_{k}, \Psi_{l}\right)_{\chi}+\mu\left(p_{k}, \Psi_{l}\right)_{\chi}=0 .
$$


On the other hand, by (3.16) and (3.13) we get that for $1 \leq l \leq k-5$,

$$
B_{\mu}\left(p_{k}, \Psi_{l}\right)=B_{\mu}\left(\Phi_{k}-\sum_{i=1}^{k-1} a_{k, i} \Phi_{i}, \Psi_{l}\right)=-a_{k, l} \rho_{l} .
$$

Hence, $a_{k, l}=0$ for $1 \leq l \leq k-5$. This means

$$
\Phi_{k}(x)=p_{k}(x)+a_{k, k-1} \Phi_{k-1}(x)+a_{k, k-2} \Phi_{k-2}(x)+a_{k, k-3} \Phi_{k-3}(x)+a_{k, k-4} \Phi_{k-4}(x) .
$$

Similarly, we deduce that

$$
\Psi_{k}(x)=q_{k}(x)+b_{k, k-1} \Psi_{k-1}(x)+b_{k, k-2} \Psi_{k-2}(x)+b_{k, k-3} \Psi_{k-3}(x) .
$$

For simplicity of the notations, we take $a_{k-1}:=a_{k, k-1}, b_{k-2}:=a_{k, k-2}, c_{k-3}:=a_{k, k-3}, d_{k-4}$ $:=a_{k, k-4}, e_{k-1}:=b_{k, k-1}, h_{k-2}:=b_{k, k-2}, m_{k-3}:=b_{k, k-3}$. Then we obtain the result (3.14).

It remains to confirm the coefficients $a_{k-1}, b_{k-2}, c_{k-3}, d_{k-4}, e_{k-1}, h_{k-2}, m_{k-3}$ and $\rho_{k}$. By (2.28), (2.26), (2.24) and (3.13) we know that

$$
\begin{aligned}
& B_{\mu}\left(\Phi_{1}, \Psi_{1}\right)=\left(\partial_{x} p_{1}, \partial_{x}\left(q_{1} \chi\right)\right)+\mu\left(p_{1}, q_{1}\right)_{\chi}=\frac{5}{16} \pi+\frac{3}{2} \mu \pi \\
& B_{\mu}\left(\Phi_{2}, \Psi_{1}\right)=\left(\partial_{x} p_{2}, \partial_{x}\left(q_{1} \chi\right)\right)+\mu\left(p_{2}, q_{1}\right)_{\chi}+a_{1} \rho_{1}=-\frac{3}{4} \pi+2 \mu \pi+a_{1} \rho_{1}=0 \\
& B_{\mu}\left(\Phi_{1}, \Psi_{2}\right)=\left(\partial_{x} p_{1}, \partial_{x}\left(q_{2} \chi\right)\right)+\mu\left(p_{1}, q_{2}\right)_{\chi}+e_{1} \rho_{1}=-\frac{9}{16} \pi+\frac{1}{2} \mu \pi+e_{1} \rho_{1}=0
\end{aligned}
$$

Hence, we have

$$
\rho_{1}=\frac{(5+24 \mu) \pi}{16}, \quad a_{1}=-\frac{(8 \mu-3) \pi}{4 \rho_{1}}, \quad e_{1}=\frac{(9-8 \mu) \pi}{16 \rho_{1}}
$$

In the same manner, we can derive the coefficients $a_{k-1}, b_{k-2}, c_{k-3}, d_{k-4}, e_{k-1}, h_{k-2}, m_{k-3}$ and $\rho_{k}$ for $k \leq 5$ as shown in (3.15).

We next verify the results in (3.15) for $k \geq 6$. In fact, by (2.28), (2.26) and (2.24) we obtain

$$
B_{\mu}\left(p_{k}, q_{k-4}\right)=\left(\partial_{x} p_{k}, \partial_{x}\left(q_{k-4} \chi\right)\right)+\mu\left(p_{k}, q_{k-4}\right)_{\chi}=-\frac{1}{32}(k-2)(k-3) \pi .
$$

On the other hand, by (3.14) and (3.13) we get

$$
\begin{aligned}
& B_{\mu}\left(p_{k}, q_{k-4}\right)=B_{\mu}\left(\Phi_{k}-a_{k-1} \Phi_{k-1}-b_{k-2} \Phi_{k-2}-c_{k-3} \Phi_{k-3}-d_{k-4} \Phi_{k-4}\right. \\
& \left.\Psi_{k-4}-e_{k-5} \Psi_{k-5}-h_{k-6} \Psi_{k-6}-m_{k-7} \Psi_{k-7}\right)=-d_{k-4} \rho_{k-4} .
\end{aligned}
$$

Therefore

$$
d_{k-4}=\frac{(k-2)(k-3) \pi}{32 \rho_{k-4}} \text {. }
$$


Similarly, we have

$$
\begin{aligned}
B_{\mu}\left(p_{k}, q_{k-3}\right) & =-c_{k-3} \rho_{k-3}+d_{k-4} e_{k-4} \rho_{k-4}=\frac{5}{32}(k-2)(k-3) \pi-\frac{1}{16} \pi \delta_{k, 4} \\
B_{\mu}\left(p_{k}, q_{k-2}\right) & =-b_{k-2} \rho_{k-2}+c_{k-3} e_{k-3} \rho_{k-3}+d_{k-4} h_{k-4} \rho_{k-4} \\
& =\frac{16 \mu \pi-3(k-4)(3 k-5) \pi}{32} \\
B_{\mu}\left(p_{k}, q_{k-1}\right) & =-a_{k-1} \rho_{k-1}+b_{k-2} e_{k-2} \rho_{k-2}+c_{k-3} h_{k-3} \rho_{k-3}+d_{k-4} m_{k-4} \rho_{k-4} \\
& =\frac{5\left(k^{2}-11 k+12\right) \pi+48 \mu \pi}{32}+\frac{3}{16} \pi \delta_{k, 2}, \\
B_{\mu}\left(p_{k}, q_{k}\right)= & \rho_{k}+a_{k-1} e_{k-1} \rho_{k-1}+b_{k-2} h_{k-2} \rho_{k-2}+c_{k-3} m_{k-3} \rho_{k-3} \\
= & \frac{5\left(k^{2}+7 k-6\right) \pi+48 \mu \pi}{32}, \\
B_{\mu}\left(p_{k}, q_{k+1}\right) & =-e_{k} \rho_{k}+a_{k-1} h_{k-1} \rho_{k-1}+b_{k-2} m_{k-2} \rho_{k-2} \\
& =\frac{16 \mu \pi-3\left(3 k^{2}+5 k-2\right) \pi}{32}, \\
B_{\mu}\left(p_{k}, q_{k+2}\right) & =-h_{k} \rho_{k}+a_{k-1} m_{k-1} \rho_{k-1}=\frac{5 k(k+1) \pi}{32} \\
B_{\mu}\left(p_{k}, q_{k+3}\right) & =-m_{k} \rho_{k}=-\frac{k(k+1) \pi}{32} .
\end{aligned}
$$

By using (3.22) and (3.21), a direct computation leads to the desired result (3.15).

Theorem 3.2. Let $u(x)$ and $u_{N}(x)$ be the solutions of (3.11) and (3.12), respectively. Then both $u(x)$ and $u_{N}(x)$ have the explicit representations in $\left\{\Phi_{k}(x)\right\}$,

$$
\begin{array}{ll}
u(x)=\sum_{k=1}^{\infty} \hat{u}_{k} \Phi_{k}(x), & u_{N}(x)=\sum_{k=1}^{N} \hat{u}_{k} \Phi_{k}(x), \\
\hat{u}_{k}=\frac{1}{\rho_{k}} B_{\mu}\left(u, \Psi_{k}\right)=\frac{1}{\rho_{k}}\left(f, \Psi_{k}\right)_{\chi}, & k \geq 1 .
\end{array}
$$

Remark 3.2. Wang and Guo [11] presented the convergence of scheme (3.12): if $\mu>\frac{14}{27}$ and $r \geq 1$, then

$$
\left\|u-u_{N}\right\|_{1, \chi} \leq c N^{1-r}\left(\sum_{k=1}^{r}\left\|(x+1)^{r / 2+k-1 / 2} \partial_{\chi}^{k} u\right\|_{\chi}^{2}\right)^{1 / 2} .
$$

\section{Numerical results}

In this section, we examine the effectiveness and accuracy of the diagonalized Chebyshev rational spectral method for solving second-order elliptic equations on the half/whole line. 


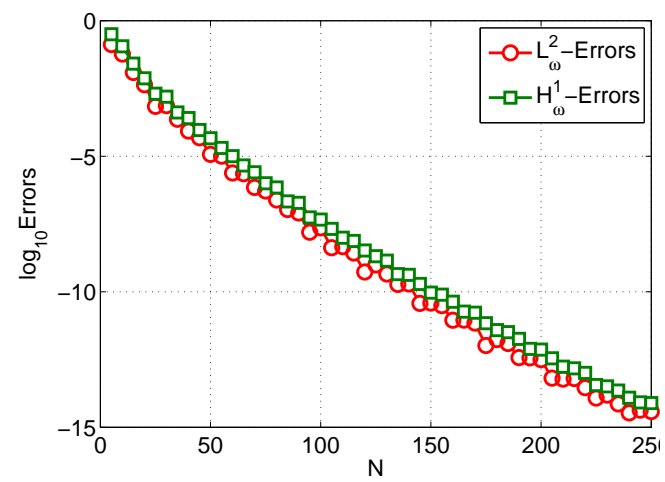

Figure 1: $u(x)=e^{-x^{2}} \sin (2 x)$.

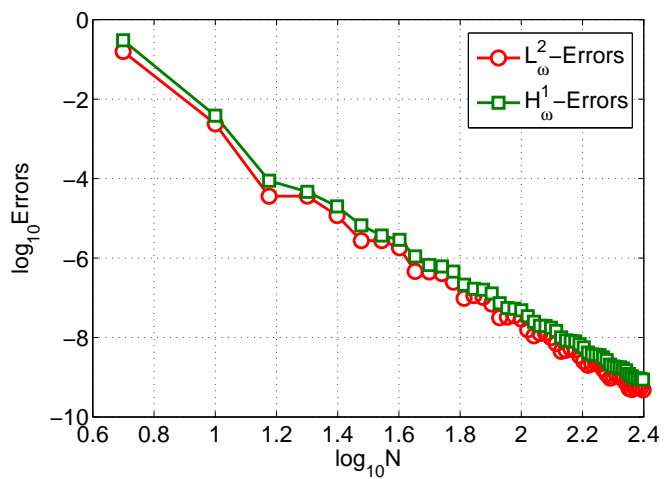

Figure 2: $u(x)=\frac{\sin (2 x)}{\left(1+x^{2}\right)^{2}}$.

We first examine the second-order elliptic problem on the whole line. We take $\mu=1$ in (3.1) and consider the following four cases:

- $u(x)=e^{-x^{2}} \sin (k x)$, which decays exponentially at infinity with oscillation. In Fig. 1, we plot the $\log _{10}$ of the discrete $L_{\omega}^{2}$ - and $H_{\omega}^{1}$ - errors vs. $N$ with $k=2$. The two near straight lines indicate an exponential convergence rate.

- $u(x)=\sin (k x)\left(1+x^{2}\right)^{-h}$, which decays algebraicly at infinity with oscillation. In Fig. 2 , we plot the $\log _{10}$ of the discrete $L_{\omega}^{2}$ - and $H_{\omega}^{1}$ - errors vs. $\log _{10} N$ with $k=h=2$. The two near straight lines indicate an algebraic convergence rate.

- $u(x)=\ln \left(1+x^{2}\right)\left(1+x^{2}\right)^{-h}$, which decays algebraicly at infinity without oscillation. In Fig. 3, we plot the $\log _{10}$ of the discrete $L_{\omega}^{2}$ - and $H_{\omega}^{1}$ - errors vs. $\log _{10} N$ with $h=3$. The two near straight lines also indicate an algebraic convergence rate.

- $u(x)=\arctan (k x)$, which does not decay at infinity. In Fig. 4, we plot the $\log _{10}$ of the discrete $L_{\omega^{-}}^{2}$ and $H_{\omega^{-}}^{1}$-errors vs. $\log _{10} N$ with $k=1$. The two near straight lines indicate again an algebraic convergence rate.

We next examine the second-order elliptic problem on the half line. We take $\mu=1$ in (3.10) and consider the following four cases:

- $u(x)=e^{-x^{2}} \sin (k x)$, which decays exponentially at infinity with oscillation. In Fig. 5, we plot the $\log _{10}$ of the discrete $L_{\chi}^{2}$ - and $H_{\chi}^{1}$ - errors vs. $N$ with $k=2$. They indicate an exponential convergence rate.

- $u(x)=\sin (k x)\left(x^{2}+1\right)^{-h}$, which decays algebraicly at infinity with oscillation. In Fig. 6, we plot the $\log _{10}$ of the discrete $L_{\chi}^{2}$ - and $H_{\chi}^{1}$ - errors vs. $\log _{10} N$ with $k=h=2$. They indicate an algebraic convergence rate.

- $u(x)=\ln \left(1+x^{2}\right)\left(1+x^{2}\right)^{-h}$, which decays algebraicly at infinity without oscillation. In Fig. 7, we plot the $\log _{10}$ of the discrete $L_{\chi}^{2}$ - and $H_{\chi}^{1}$ - errors vs. $\log _{10} N$ with $h=2$. They also indicate an algebraic convergence rate. 


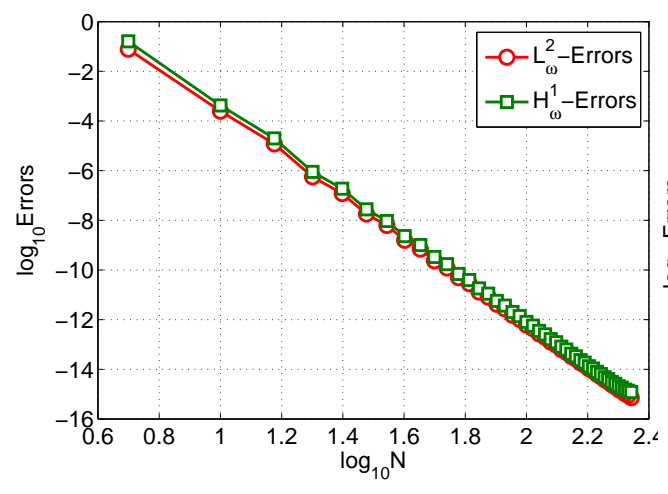

Figure 3: $u(x)=\frac{\ln \left(1+x^{2}\right)}{\left(1+x^{2}\right)^{3}}$.

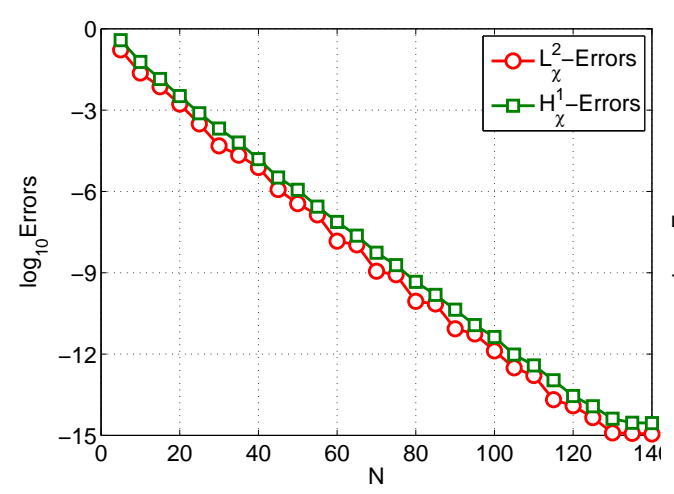

Figure 5: $u(x)=e^{-x^{2}} \sin (2 x)$.

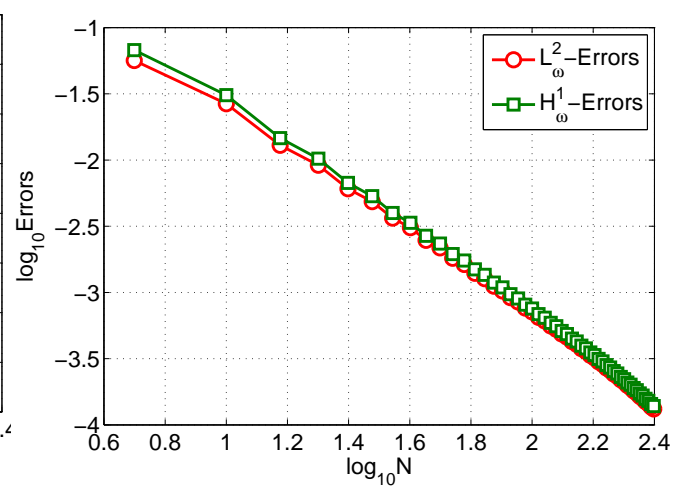

Figure 4: $u(x)=\arctan (x)$.

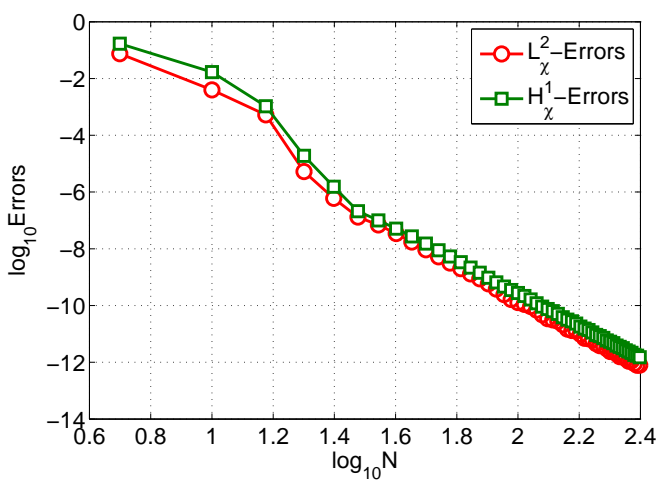

Figure 6: $u(x)=\frac{\sin (2 x)}{\left(x^{2}+1\right)^{2}}$.

- $u(x)=\arctan (k x)$, which does not decay at infinity. In Fig. 8, we plot the $\log _{10}$ of the discrete $L_{\chi}^{2}$ - and $H_{\chi}^{1}$ - errors vs. $\log _{10} N$ with $k=1$. They indicate again an algebraic convergence rate.

To demonstrate the essential superiority of our diagonalized Chebyshev rational spectral method to the classic Chebyshev rational spectral method, we examine the issue on condition numbers for the resulting algebraic systems.

The diagonalized Chebyshev rational spectral method use the Sobolev bi-orthogonal Chebyshev rational functions $\left\{\frac{\varphi_{k}(x)}{\sqrt{\eta_{k}}}, \frac{\psi_{k}(x)}{\sqrt{\eta_{k}}}\right\}_{k=0}^{N}$ and $\left\{\frac{\Phi_{k}(x)}{\sqrt{\rho_{k}}}, \frac{\Psi_{k}(x)}{\sqrt{\rho_{k}}}\right\}_{k=1}^{N}$ as the basis functions for (3.1) and (3.10), respectively. All the condition numbers of the corresponding total stiff matrices are equal to 1 . While in the classic Chebyshev rational spectral method, the basis functions are chosen as $\left\{R_{k}(x)\right\}_{k=0}^{N}$ and $\left\{r_{k}(x)+r_{k-1}(x)\right\}_{k=1}^{N}$ for (3.1) and (3.10), respectively. The corresponding total stiff matrices have off-diagonal entries. In Table 4.1 below, we take $\mu=1$ and list the condition numbers of the total stiff matrices of the classic Chebyshev rational spectral method for (3.1) and (3.10). We note that the condition numbers of the resulting systems increase asymptotically as $\mathscr{O}\left(N^{2}\right)$. 


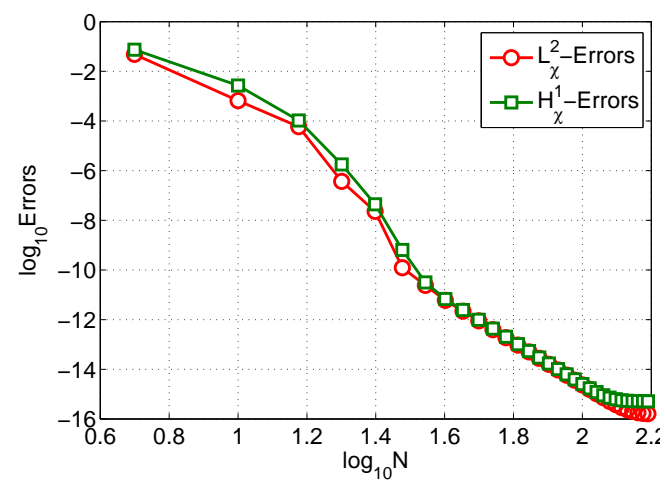

Figure 7: $u=\frac{\ln \left(1+x^{2}\right)}{\left(1+x^{2}\right)^{2}}$.

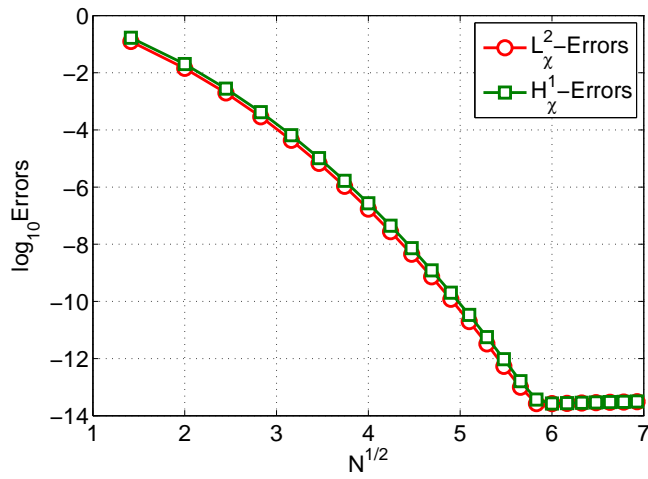

Figure 8: $u(x)=\arctan (x)$.

Table 1: Condition numbers of the classic Chebyshev rational spectral method.

\begin{tabular}{||c|c|c|c|c|c|c||}
\hline & $N=40$ & $N=80$ & $N=120$ & $N=160$ & $N=200$ & $N=240$ \\
\hline Eq. (3.1) & $1.1288 \mathrm{e}+03$ & $5.1173 \mathrm{e}+03$ & $1.2125 \mathrm{e}+04$ & $2.2199 \mathrm{e}+04$ & $3.5367 \mathrm{e}+04$ & $5.1644 \mathrm{e}+04$ \\
\hline Eq. (3.10) & $1.8147 \mathrm{e}+03$ & $7.6508 \mathrm{e}+03$ & $1.7578 \mathrm{e}+04$ & $3.1615 \mathrm{e}+04$ & $4.9769 \mathrm{e}+04$ & $7.2045 \mathrm{e}+04$ \\
\hline
\end{tabular}

Acknowledgments This work was supported in part by National Natural Science Foundation of China (Nos. 11571238 and 11601332).

\section{References}

[1] M. Azaiez, J. Shen, C. J. Xu And Q. Q. Zhuang, A Laguerre-Legendre spectral method for the Stokes problem in a semi-infinite channel, SIAM J. Numer. Anal., 47(9) (2008), pp. 271-292.

[2] C. Bernardi And Y. MAdAY, Spectral Methods of Numerical Analysis, Elsevier, Amsterdam, 1997.

[3] J. P. BoyD, Spectral methods using rational basis functions on an infinite interval, J. Comput. Phys., 69 (1987), pp. 112-142.

[4] J. P. Boyd, Orthogonal rational functions on a semi-infinite interval, J. Comput. Phys., 70 (1987), pp. 63-88.

[5] C. Canuto, M. Y. Hussaini, A. Quarteroni and T. A. Zang, Spectral Methods, Fundamentals in Single Domains, Springer, Berlin, 2006.

[6] C. I. Christov, A complete orthogonal system of functions in $l^{2}(-\infty, \infty)$ space, SIAM J. Appl. Math., 42 (1982), pp. 1337-1344.

[7] L. Fernandez, F. Marcellan, T. Pérez, M. Piñar and X. Yuan, Sobolev orthogonal polynomials on product domains, J. Comp. Anal. Appl., 284 (2015), pp. 202-215.

[8] D. Funaro, Polynomial Approximation of Differential Equations, Springer-Verlag, Berlin, 1992.

[9] B. Y. Guo AND J. SHEN, Laguerre-Galerkin method for nonlinear partial differential equations on a semi-infinite interval, Numer. Math., 86 (2000), pp. 635-654.

[10] B. Y. Guo, J. SHEN AND Z. Q. WANG, A rational approximation and its applications to differential equations on the half line, J. Sci. Comput., 15 (2000), pp. 117-147.

[11] B. Y. GuO, J. SHEN AND Z. Q. WANG, Chebyshev rational spectral and pseudospectral methods on a semi-infinite interval, Internat. J. Numer. Methods Engrg., 53 (2002), pp. 65-84. 
[12] B. Y. Guo, L. L. WANG AND Z. Q. WANG, Generalized Laguerre interpolation and pseudospectral method for unbounded domains, SIAM J. Numer. Anal., 43 (2006), pp. 2567-2589.

[13] F. J. LiU, H. Y. Li AND Z. Q. WANG, Spectral methods using generalized Laguerre functions for second and fourth order problems, Numer. Algor., 75 (2017), pp. 1005-1040.

[14] F. J. LiU, Z. Q. WANG AND H. Y. LI, A fully diagonalized spectral method using generalized Laguerre functions on the half line, Adv. Comput. Math., 43 (2017), pp. 1227-1259.

[15] H. P. MA AND B. Y. Guo, Composite Legendre-Laguerre pseudospectral approximation in unbounded domains, IMA J. Numer. Anal., 21 (2001), pp. 587-602.

[16] H. P. MA, W. W. Sun AND T. TANG, Hermite spectral methods with a time-dependent scaling for parabolic equations in unbounded domains, SIAM J. Numer. Anal., 43 (2005), pp. 58-75.

[17] F. Marcellán AND Y. Xu, On Sobolev orthogonal polynomials, Expo. Math., 33 (2015), pp. 308352.

[18] T. Matsushima and P. Marcus, A spectral method for unbounded domains, J. Comput. Phys., 137 (1997), pp. 321-345.

[19] J. SHen, Stable and efficient spectral methods in unbounded domains using Laguerre functions, SIAM J. Numer. Anal., 38 (2000), pp. 1113-1133.

[20] J. Shen, T. TAng And L. L. WAng, Spectral Methods: Algorithms, Analysis and Applications, Springer-Verlag, Berlin, 2011.

[21] J. Shen AND L. L. WANG, Fourierization of the Legendre-Galerkin method and a new space-time spectral method, Appl. Numer. Math., 57 (2007), pp. 710-720.

[22] Z. Q. WANG AND B. Y. GUO, A rational approximation and its applications to nonlinear partial differential equations on the whole line, J. Math. Anal. Appl., 274 (2002), pp. 374-403.

[23] Z. Q. WANG AND B. Y. GuO, Jacobi rational approximation and spectral method for differential equations of degenerate type, Math. Comp., 77 (2008), pp. 883-907.

[24] X. M. XIANG AND Z. Q. WANG, Generalized Hermite spectral method and its applications to problems in unbounded domains, SIAM J. Numer. Anal., 48 (2010), pp. 1231-1253. 